\title{
Molecular Dynamics Study of the Dependence of Water Solvation Free Energy on Solute Curvature and Surface Area
}

\author{
A. Wallqvist ${ }^{\dagger}$ and B. J. Berne* \\ Department of Chemistry and Center for Biomolecular Simulation, Columbia University, \\ New York, New York 10027 \\ Received: August 5, $1994^{\otimes}$
}

We have investigated changes in water properties when deforming an initially spherical cavity into an oblate ellipsoid of equal volume in liquid water. The purely hydrophobic cavity has an initial thermal radius of $6.45 \AA$ and is flattened out to an oblate ellipse with a thickness corresponding to one layer of methane molecules. The water-solute interactions are modeled by a repulsive, single-site Gay-Berne potential that preserves the volume of the solute; water-water interactions are modeled using a pairwise additive potential. The Gibbs free energy change, $\Delta G$, of the aqueous solution was calculated using thermodynamic perturbation theory. Comparison with the process of radially expanding a sphere shows that the free energy change cannot consistently be interpreted as being solely proportional to an exposed solute area but contains terms involving the curvature of the solute. As the meanings of exposed surface area and molecular curvature are not welldefined concepts on the microscopic length scale, these terms have to be defined to yield a consistent interpretation of the free energy data. The microscopic origin of the curvature dependence of the free energy is traced back to changes in water-water interactions in the region immediately surrounding the solute. The process of deforming the liquid around the hydrophobic pocket was found to be dominated by entropic contributions. The free energy values do not contain any contributions arising from the deformation of the hydrophobic solute itself nor any attractive solute-solvent term and thus cannot be compared directly with hydrocarbon-water surface tension data.

\section{Introduction}

The hydrophobic solvation free energy of an arbitrary molecular moiety has often been approximated by the product of a solvation free energy per unit surface area and the exposed surface area of the molecule. ${ }^{1}$ In a particular parametrization, this area is calculated by rolling a sphere of radius $1.4 \AA^{2}$ on the solute molecule ${ }^{2}$ and can be further corrected by the introduction of a curvature-dependent term. ${ }^{3-5}$ With this procedure the value for the free energy per $\AA^{2}$ has been determined from thermodynamic data on alkane solubilities, and a close correspondence to the macroscopic hydrocarbon-water surface tension has been found. Such attempts to reconcile the macroscopic surface tension with microscopic theories of cavity formation ${ }^{4-6}$ have been criticized, due to the lack of a theoretical basis for connecting the macroscopic and microscopic concept of surface tension as well as for the volume correction terms used to reevaluate the alkane solubility data. ${ }^{7-9}$

It is thus of considerable interest to use computer experiments to explore the free energy of hydration of solutes with arbitrary surfaces. The structure and equilibrium properties of water in the hydration shell of small hydrophobic solute molecules ${ }^{10-14}$ as well as at more extended hydrophobic surfaces ${ }^{15-19}$ have been studied in detail. Thermodynamic perturbation methods have also been used in order to calculate the free energy of hydration of small solutes. ${ }^{20,21}$

In this paper we simulate two different solute-water systems using constant-pressure molecular dynamics using the newly devised restricted effective representation (RER) model of water which incorporates effective liquid state charges in a pairwise additive potential, RER(pair). ${ }^{22}$ In one case we calculate the

\footnotetext{
${ }^{\dagger}$ Present address: PRI/NCI-FCRDC, Building 1052, Room 238, P.O. Box B, Frederick, MD 21702.

${ }^{\otimes}$ Abstract published in Advance ACS Abstracts, February 1, 1995.
}

change in Gibbs free energy $\Delta G$ accompanying the deformation of a large sphere into an oblate ellipsoid of increasing eccentricity and indeed find that for large eccentricities $\Delta G$ becomes a linear increasing function of surface area, although it is not linear through the whole domain. The slope is found to be considerably larger than the gas-liquid surface tension for the model of water used in these simulations. The gas-liquid surface tension for this water model was determined in a separate molecular dynamics simulation of the equilibrium interface between liquid water and its vapor. ${ }^{23}$ In the other solute-water system, we simulate the free energy change accompanying the growth of a spherical particle starting at the same sphere size used in the deformation study. Again, the $\Delta G$ is found to depend linearly on the surface area albeit with approximately double the slope of $\Delta G$ versus $\Delta A$ found in the deformation simulation. In addition neither slope is the same as the gasliquid surface tension. This difference shows that one cannot ignore shape effects of the cavity on the free energy. The data for both the deformed sphere and the expanding sphere are found to be fit well by an expression of the following form:

$$
\Delta G(\bar{R} \mathcal{A})=\lambda \Delta \bar{R}+\gamma \Delta \mathcal{A}
$$

where $\bar{R}$ is the average radius of curvature and $\mathcal{A}$ denotes the area. The coefficient $\gamma$ is still significantly larger than the gasliquid surface tension. This indicates that the structure of the water at the interface is much sharper than at the gas-liquid interface. These experiments call into question the proposal that water structure at the interface between a hard sphere and water is very similar to the gas-liquid interface..$^{24,25}$

\section{Model System}

The interaction between an idealized oblate, hydrophobic ellipsoid and a water molecule is defined in such a way as to 
allow us to continuously vary the shape of the hydrophobic object from a sphere of radius, $\sigma_{0}$, to an oblate ellipsoid corresponding roughly to a sheet of methane molecules. In this deformation process the solute surface area increases but its volume remains constant, thus enabling us to study the isolated effect of shape changes on the free energy of solution. Since we are primarily interested in the solvent response to the hydrophobic object, we have chosen to use a purely repulsive solute-water interaction, modeled as the repulsive part of the Lennard-Jones interaction in the Gay-Berne potential, ${ }^{26,27}$ for the interaction between an ellipsoidal particle and a spherical particle

$$
U_{\mathrm{sw}}=k T\left(\frac{\sigma_{0}}{r-\sigma(\chi ; v)+\sigma_{0}}\right)^{12}
$$

where $k T$ is the thermal energy, $\sigma(\chi ; \nu)$ is an orientationdependent Lennard-Jones diameter,

$$
\sigma(\chi ; v)=\frac{\sigma_{\perp}}{\left(1-\chi \cos ^{2} v\right)^{1 / 2}}
$$

where the anisotropy parameter $\chi$ is given by

$$
\chi=\left(\sigma_{\|}^{2}-\sigma_{\perp}^{2}\right) / \sigma_{\|}^{2}
$$

and where the angle between the orientation of the major axis of the ellipsoid, $\hat{\mathbf{u}}$, and the unit vector pointing from the center of the ellipsoid to the oxygen atom of the water molecule, $\hat{\mathbf{r}}$, is

$$
v=\arccos (\hat{\mathbf{r}} \hat{\mathbf{u}})
$$

The length of the oblate ellipse along the major, $\sigma_{\| 1}=L \sinh u$, and minor, $\sigma_{\perp}=L \cosh u$, axes is determined from

$$
L[u(e)]^{3}=\frac{\sigma_{0}^{3}}{\cosh ^{2}[u(e)] \sinh [u(e)]}
$$

and

$$
u(e)=\frac{1}{2} \ln \left(-\left[\frac{1+\sqrt{1-e^{2}}}{e}\right]\right)
$$

together with

$$
e=\frac{\sigma_{\|}^{2}-\sigma_{\perp}^{2}}{\sigma_{\|}^{2}+\sigma_{\perp}^{2}}=\chi \frac{\sigma_{\|}^{2}}{\sigma_{\|}^{2}+\sigma_{\perp}^{2}}
$$

At an eccentricity $e=0$,

$$
\sigma(0 ; \nu)=\sigma_{0}
$$

and the model reduces to the repulsive Lennard-Jones interaction between a spherical solute and a water molecule.

Thus, the potential is described by two parameters, $\chi$ or $e$, denoting the eccentricity of the ellipsoid and the radius of the underlying undeformed spherical object, $\sigma_{0}$. The volume constraint that is satisfied by the ellipsoid is

$$
\frac{4 \pi \sigma_{0}^{3}}{3}=\frac{4 \pi \sigma_{\perp}^{2} \sigma_{\| 1}}{3}
$$

The van der Waals area, ${ }^{28}$ defined by the hydrophobic solutewater interaction traced out by $r=\sigma(\chi ; \nu)$, is given by

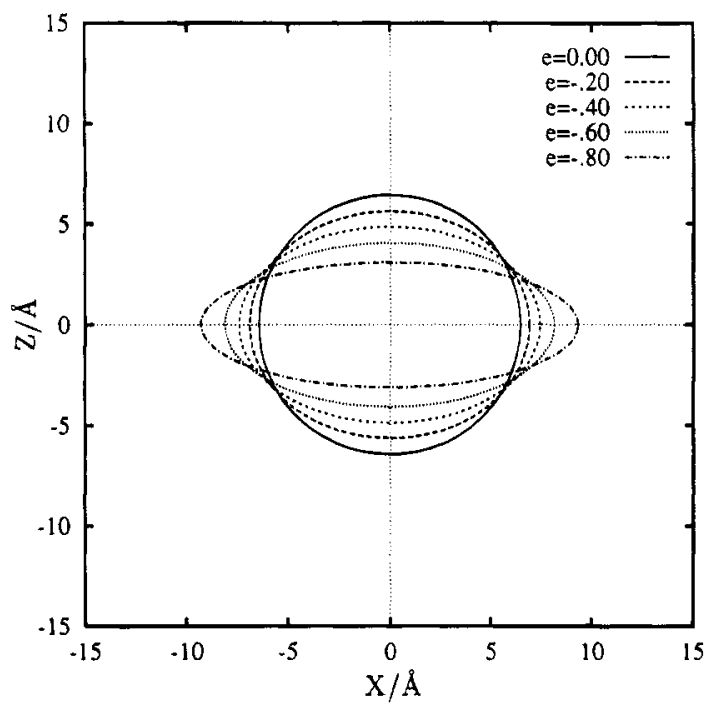

Figure 1. A cut through the equipotential surface, $U_{\mathrm{sw}}=k T$, for various values of the eccentricity, $e$. The unit cell used in the simulation is drawn for comparison.

TABLE 1: Geometrical System Characteristics

\begin{tabular}{rccccc}
\hline$e$ & $\bar{R}, \AA$ & $\tau_{\text {vdw }}, \AA^{2}$ & $\sigma_{\perp}, \AA$ & $\sigma_{\| l,} \AA$ & $V_{\text {solute }}, \AA^{3}$ \\
\hline \multicolumn{5}{c}{ Sphere Deformation into an Ellipse } \\
0.0 & 6.450 & 522.8 & 6.450 & 6.450 & 1124 \\
-0.1 & 6.461 & 523.7 & 6.033 & 6.669 & 1124 \\
-0.2 & 6.496 & 526.7 & 5.635 & 6.901 & 1124 \\
-0.3 & 6.555 & 532.1 & 5.247 & 7.151 & 1124 \\
-0.4 & 6.643 & 540.6 & 4.863 & 7.428 & 1124 \\
-0.5 & 6.768 & 553.4 & 4.472 & 7.746 & 1124 \\
-0.6 & 6.945 & 572.7 & 4.063 & 8.126 & 1124 \\
-0.7 & 7.205 & 603.1 & 3.618 & 8.612 & 1124 \\
-0.8 & 7.623 & 656.7 & 3.101 & 9.303 & 1124 \\
\multicolumn{5}{c}{ Sphere Growth } \\
0.0 & 6.450 & 522.8 & 6.450 & 6.450 & 1124 \\
0.0 & 6.650 & 555.7 & 6.650 & 6.650 & 1232 \\
0.0 & 6.850 & 589.6 & 6.850 & 6.850 & 1346 \\
0.0 & 7.050 & 624.6 & 7.050 & 7.050 & 1468 \\
0.0 & 7.250 & 660.5 & 7.250 & 7.250 & 1596
\end{tabular}

$$
\mathscr{N}^{\mathrm{dW}}=2 \pi \sigma_{\perp}^{2}\left[1+\frac{1-\chi^{2}}{2 \chi} \ln \left(\frac{1+\chi}{1-\chi}\right)\right]
$$

The van der Waals area corresponds to the $k T$ equipotential surface of water-solute interactions. The average curvature of this surface is given by

$$
\bar{R}^{\mathrm{vdW}}=\frac{\sigma_{\| 1}}{2}\left[1+\frac{\arcsin \chi}{\chi \sqrt{1-\chi^{2}}}\right]
$$

We have chosen a system that is large enough to superficially mimic that of a small micelle, with $\sigma_{0}$ set to $6.45 \AA$. Even though the range of $\chi$ can extend from $0 \rightarrow-\infty$ for an oblate ellipsoid, we have restricted the range so that the most extended shape is no thinner than one layer of methane molecules. For a thinner object, water-water interactions above and below the extended surface would give unphysical results. This restriction gives the range of $e$ that we are interested in as $0 \rightarrow-0.8$. Thus, the most extended oblate shape has $\sigma_{\perp}=3.1 \AA$ and $\sigma_{\|}=$ $9.3 \AA$. In order to achieve full hydration for the extended oblateshaped hydrophobic solute, we use a simulation box of length $31 \AA$. The hydrophobic object thus takes up $4 \%$ of the total volume of the simulation cell. In Figure 1 we plot some equipotential curves of the water-ellipsoid interaction model for different values of the eccentricity. In Table 1 some geo- 
TABLE 2: RER(pair) Water-Water Potential Parameters ${ }^{a}$

\begin{tabular}{cccccccc}
\hline$q_{\mathrm{o}}, e$ & $q_{\mathrm{H}}, e$ & $C_{12}, \mathrm{~kJ} /\left(\mathrm{mol} \AA^{12}\right)$ & $C_{6}, \mathrm{~kJ} /\left(\mathrm{mol} \AA^{6}\right)$ & $C_{4}, \mathrm{~kJ} /\left(\mathrm{mol} \AA^{4}\right)$ & $C_{5}, \mathrm{~kJ} / \mathrm{mol}$ & $w_{\mathrm{t}}, \AA^{-2}$ & $r_{\mathrm{t}}, \AA$ \\
\hline-0.920 & 0.460 & 3500000 & -3100 & 15.0 & -1.000 & 1.5
\end{tabular}

a The monomer geometry of the water molecules is given by a bond length of $r_{0}=0.96 \AA$ and a bond angle of $\theta_{0}=104.52^{\circ}$. In order to recover the potential energy in $\mathrm{kJ} / \mathrm{mol}$ when using eq $20,1 / 4 \pi \epsilon_{0}$ should be set to 1389.0 . The correction term to be added to the potential due to the self-energy of the dipole moment, $V_{\text {self, }}$ is $13.2 \mathrm{~kJ} / \mathrm{mol}$ at $300 \mathrm{~K}$.

metrical properties of the system in terms of the van der Waals area and curvature are given.

\section{Free Energy Calculations}

Thermodynamic and statistical perturbation theory originally due to Zwanzig ${ }^{29}$ can be used to calculate relative and absolute free energies for molecularly detailed systems (see for example the review by Beveridge and DiCapua ${ }^{30}$ ). The Gibbs free energy of a system with $N$ particles at a given temperature, $T$, and pressure, $P$, is given by

$$
G_{0}=-k T \ln \Delta_{0}
$$

where $k$ is Boltzmann's constant, $\Delta_{0}$ is the partition function for the isobaric ensemble, written as a classical phase space integral over the coordinates, $q$, momenta, $p$, and volume, $v$, of the reference Hamiltonian, $\mathscr{K}_{0}$,

$$
\Delta_{0}=\frac{1}{N ! h^{3 N}} \int \mathrm{d} v \mathrm{~d} p \mathrm{~d} q \mathrm{e}^{-\left(\mathscr{R}_{0}+P v\right) / k T}
$$

and $h$ is Planck's constant. We now introduce another Hamiltonian, $\mathscr{G}$,

$$
\mathscr{H}_{1}=\mathscr{K}_{0}+\Delta V
$$

where $\Delta V$ is a perturbation of the Hamiltonian $\mathscr{F}_{0}$. Then the change in free energy arising from the transformations $\mathscr{H}_{0} \rightarrow$ $\mathscr{F}_{1}$ can be written as

$$
\Delta G=G_{1}-G_{0}=-k T \ln \left\{\frac{\int \mathrm{d} v \mathrm{~d} p \mathrm{~d} q \mathrm{e}^{-\Delta V / k T} \mathrm{e}^{-\left(\mathscr{H}_{0}+P v\right) / k T}}{\int \mathrm{d} v \mathrm{~d} p \mathrm{~d} q \mathrm{e}^{-\left(\mathscr{F}_{0}+P v\right) / k T}}\right\}
$$

which is equivalent to averaging the exponential of the perturbation, $\Delta V$, in the reference system, i.e.,

$$
\Delta G=-k T \ln \left\langle\mathrm{e}^{-\Delta V / k T}\right\rangle_{0}
$$

The entropy change in the system can be evaluated from the temperature derivative of the Gibbs free energy,

$$
\left(\frac{\partial \Delta G}{\partial T}\right)_{P}=-\Delta S
$$

Taking the temperature derivative of eq 16 and multiplying by $T$ yields an expression for the entropic contribution to the free energy, ${ }^{20,31}$

$$
-T \Delta S=\Delta G-\frac{\left\langle\mathscr{F}_{1} \mathrm{e}^{-\Delta V / k T}\right\rangle_{0}}{\left\langle\mathrm{e}^{-\Delta V / k T}\right\rangle_{0}}+\left\langle\mathscr{H}_{0}\right\rangle_{0}
$$

\section{Computational Procedures}

In the simulation of the liquid water/solute system we used 936 water molecules in order to fully hydrate the deformable hydrophobic solute. In order to reproduce liquidlike densities for the bulk aqueous solution, this system was enclosed in a periodically replicated cubic box with a side of $31.0 \AA$. For water-water interactions we have employed the RER model of water, which incorporates effective liquid state charges in a pairwise additive potential, RER(pair). ${ }^{22}$ The effective pair potential model is assigned a permanent dipole moment of 2.60 D. The functional choice of the pair potential portion of the water-water potentials is

$$
\begin{array}{r}
V_{\text {pair }}\left(\left\{\mathbf{R}_{i}\right\},\left\{\mathbf{R}_{j}\right\}\right)=\frac{C_{12}}{r_{\mathrm{oo}}{ }^{12}}+\frac{C_{6}}{r_{\mathrm{oo}}{ }^{6}}+\frac{C_{4}}{r_{\mathrm{oo}}{ }^{4}}+C_{\mathrm{t}} \mathrm{e}^{-w_{\mathrm{t}}\left(r_{\mathrm{oo}}-r_{\mathrm{t}}\right)^{2}}+ \\
\sum_{\alpha \in \mathrm{i}, \beta \in \mathrm{j}}^{\text {atoms }} \frac{q_{\alpha} q_{\beta}}{4 \pi \epsilon_{0} r_{\alpha \beta}}
\end{array}
$$

where $\left\{\mathbf{R}_{i}\right\}$ denotes the coordinates of water molecule $i, r_{\mathrm{oo}}$ is the distance between oxygen atoms on water molecules $i$ and $j$, and $r_{\alpha \beta}$ is the radial distance between two atoms on the $i$ th and $j$ th water molecule. The total potential energy must be parametrized for the polarization energy required to change the charges from gas to liquid state charges, $V_{\text {self. }}{ }^{22,32,33}$ The parameter values are given in Table 2 .

The equations of motion were integrated using the Rattle version $^{34}$ of the velocity Verlet algorithm ${ }^{35}$ in order to maintain the internal bond lengths and bond angle constraints of the rigid molecule. The time step was set to $2.0 \mathrm{fs}$. The hydrophobic solute was not allowed to move but was kept fixed at the box center. Temperatures were maintained at room temperature by periodically rescaling the velocities. A constant pressure molecular dynamics algorithm was used to maintain the pressure at 1 bar. ${ }^{35}$ Both the translational and rotational temperatures were monitored so as to avoid a temperature imbalance between these degrees of freedom. All interactions were spherically truncated at half the box length, which has been shown to be adequate in order to account for the long-range interactions in most water properties. ${ }^{36}$

Two sets of simulations were performed, one in which a spherical solute-water interaction was transformed into an oblate ellipsoidal surface and one in which the spherical symmetry was maintained but with an increase in radius. For the ellipsoidal case a set of simulations between $e=0.0$ and $e$ $=-0.8$ was carried out in order to characterize the free energy change according to eq 17 . The eccentricity, $e$, was changed in steps of -0.1 for the reference states, and the free energy changes were accumulated in intervals of \pm 0.02 for each reference state. In the case of the expansion of the sphere, the solute-water cavity, $\sigma(0 ; v)$, was changed between 6.45 and $7.25 \AA$ in steps of $0.20 \AA$. Each reference system was equilibrated for $10.0 \mathrm{ps}$, starting from the last configuration of the preceding size. Thermodynamic averages were then calculated by block averaging five simulations of $5.0 \mathrm{ps}$ each. The error in the free energy for the perturbed systems was estimated as twice the standard deviation obtained from the block averaging procedure,

$$
\text { error in } X= \pm 2 \sqrt{\frac{\sum_{n=1}^{N}\left(X_{i}-X_{\mathrm{ave}}\right)^{2}}{N(N-1)}}
$$

where $X$ is the estimated property, $X_{\text {ave }}$ is the average, and $N$ is the number of block averaged systems. As the perturbation 


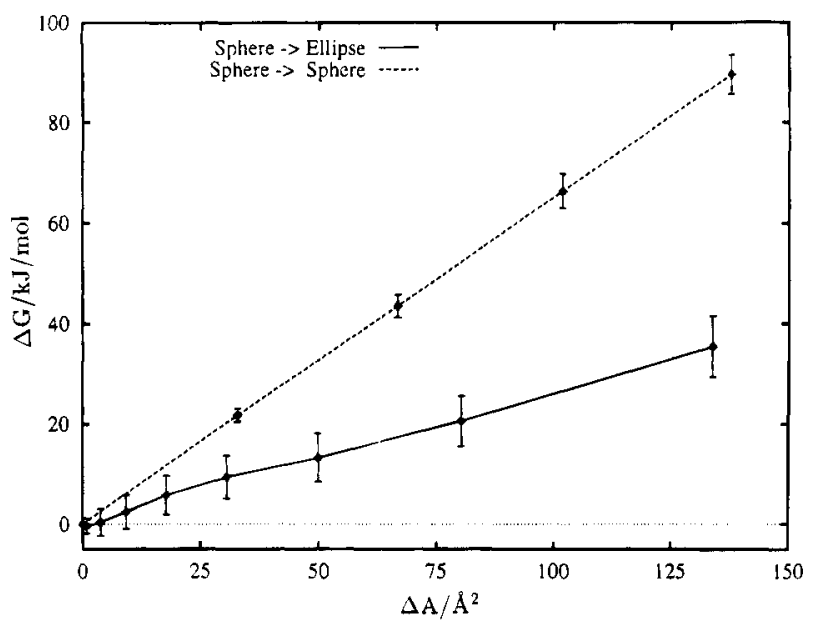

Figure 2. Gibbs free energy change, $\Delta G$, as a function of the van der Waals area, $\mathcal{A}^{\mathrm{dW}}$, for the processes of deforming a sphere into an ellipsoid and for radially expanding a sphere. The choice of the van der Waals area is an arbitrary choice.

TABLE 3: Free Energies

\begin{tabular}{|c|c|c|c|}
\hline$e$ & $\bar{R}, \AA$ & $\Delta G, \mathrm{~kJ} / \mathrm{mol}$ & $-T \Delta S, \mathrm{~kJ} / \mathrm{mol}$ \\
\hline $\begin{array}{r}0.0 \\
-0.1 \\
-0.2 \\
-0.3 \\
-0.4 \\
-0.5 \\
-0.6 \\
-0.7 \\
-0.8\end{array}$ & $\begin{array}{l}\text { Sphere } \\
6.450 \\
6.461 \\
6.496 \\
6.555 \\
6.643 \\
6.768 \\
6.945 \\
7.205 \\
7.623\end{array}$ & $\begin{array}{r}\text { mation into as } \\
0.0 \pm 0.0 \\
-0.4 \pm 1.5 \\
0.4 \pm 2.7 \\
2.4 \pm 3.4 \\
5.8 \pm 3.9 \\
9.4 \pm 4.3 \\
13.3 \pm 4.8 \\
20.6 \pm 5.0 \\
35.5 \pm 6.1\end{array}$ & $\begin{array}{r}0.0 \pm 0.0 \\
-2.1 \pm 4.6 \\
-3.4 \pm 8.4 \\
0.9 \pm 9.3 \\
7.4 \pm 10.6 \\
13.3 \pm 11.4 \\
18.7 \pm 12.3 \\
30.4 \pm 15.7 \\
60.5 \pm 17.2\end{array}$ \\
\hline $\begin{array}{l}0.0 \\
0.0 \\
0.0 \\
0.0 \\
0.0\end{array}$ & $\begin{array}{l}6.450 \\
6.650 \\
6.850 \\
7.050 \\
7.250\end{array}$ & $\begin{array}{r}\text { rere Growth } \\
0.0 \pm 0.0 \\
21.7 \pm 1.3 \\
43.5 \pm 2.3 \\
66.4 \pm 3.4 \\
89.6 \pm 3.9\end{array}$ & $\begin{array}{r}0.0 \pm 0.0 \\
38.1 \pm 4.7 \\
70.8 \pm 6.0 \\
107.2 \pm 8.2 \\
143.1 \pm 9.5\end{array}$ \\
\hline
\end{tabular}

technique estimates the change in free energy relative to each reference system simulated, the total free energy change has to be reconstructed by connecting the individual free energy curves from each reference system. The error estimate of the free energy curve is then propagated using the error of the connecting point. ${ }^{20,37}$

Although the free energy perturbations are large on an absolute scale, these values are much smaller when considering the uniform nature of the perturbation. Thus, the change per surface water molecule is still small and essentially uniform over the entire surface.

\section{Thermodynamic Properties}

The calculated free energy changes with propagated error bars are given in Table 3 and Figure 2 as a function of the change in the van der Waals area for both systems. The free energy penalty increases as the solute is deformed away from the spherical shape. The large size of the solute precludes any ordered clathrate hydrate structure, which is otherwise typical for small nonpolar solutes, and the free energy change appears smooth. The free energy change has a large entropic contribution, as calculated from eq 19 and given in Table 3. The main component of the free energy change is thus entropic, consistent with the general view of solvation of large nonpolar molecules. The unfavorable entropy may be a consequence of the reduced volume available to a water molecule at the surface rather then arising from a more ordered structure of the water solution.

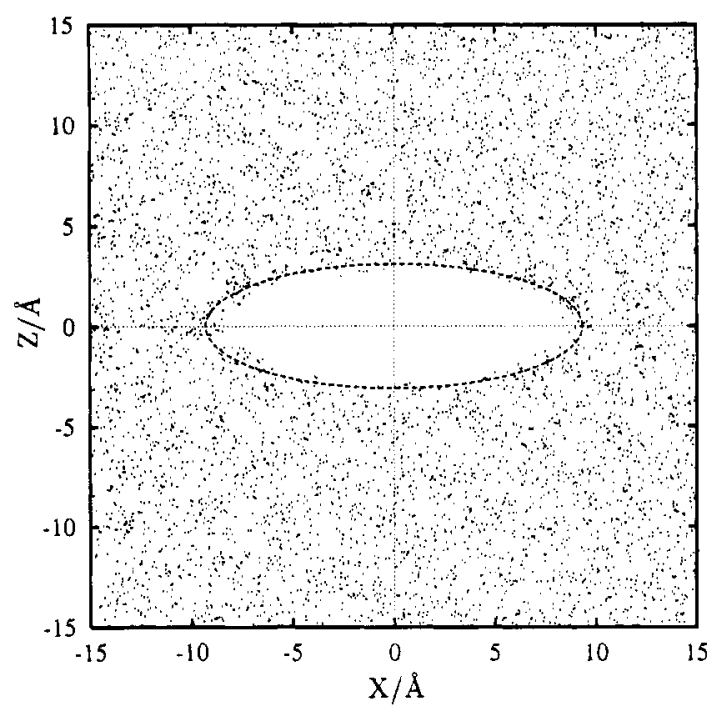

Figure 3. A coordinate projection of 25 water configurations with a hydrophobic solute $(e=-0.8)$ present. The positions of water oxygen atoms are plotted and give an illusion of a smeared 2D distribution. Water molecules obscuring the solute have been removed, i.e., those waters whose $y$ coordinate is greater than $|4.0| \AA$. The apparent crowding of water molecules at the ends of the ellipsoid is an artifact of the projection. The equipotential surface for the water-solute interactions is indicated by the line $r=\sigma(\chi ; v)$, i.e., for $U_{\mathrm{sw}}=k T$.

The van der Waals area given in Table 1 corresponds to the $k T$ equipotential surface of water-ellipsoid interaction. If we look at coordinate projections of the water configurations around the most eccentric oblate ellipsoid, we get an idea of the shape of the solute cavity. In Figure 3 we have generated a crosssectional picture of the oblate water cavity by removing the obscuring water molecules in front of and in back of the solute. The surrounding water cavity has the correct oblate shape, but local ordering of the surface waters with respect to the hydrogenbonded bulk liquid prevents a completely smooth water cavity. The indicated van der Waals surface is only one possible definition of the solute area. However one defines the microscopic surface area, it should reflect the resulting local ordering at the solute/solvent interface..$^{38-40}$ This property of the interface is not yet experimentally observable, and thus detailed knowledge can be found only from a computer simulation.

From Figure 2 we conclude that the free energy change of the sphere growth exhibits a linear relationship between free energy change and area change. The slope gives an effective interfacial surface tension coefficient, $\gamma_{\mathrm{sw}}^{\mathrm{vdW}}=1.0 \times 10^{2} \mathrm{dyn} /$ $\mathrm{cm}$, between our model system and the hydrophobic solute. The data from the sphere deformation simulations are noisier but can also be interpreted as having an approximate linear relationship between free energy and area, yielding a $\gamma_{\mathrm{sw}}^{\mathrm{vdW}}=$ $0.4 \times 10^{2} \mathrm{dyn} / \mathrm{cm}$. No reasonable redefinition of the surface area brings these two free energy coefficients into accord. From this plot we conclude that the free energy of an oblate ellipsoid is smaller than that of a sphere of the same surface area. Thus, for the simple system considered here oblate ellipsoids will be more soluble in water than spheres of the same surface area.

The reverisble work, $\Delta G$, required to deform the surface as a function of the change in the average radius of curvature $\bar{R}$ (cf. eq 12) and area $\mathcal{A}$ can be written as ${ }^{41}$

$$
\Delta G(\bar{R}, \mathcal{A})=\lambda \Delta \bar{R}+\gamma \Delta \mathcal{L}
$$

where $\lambda$ and $\gamma$ are constants. The coefficient $\gamma$ has the same units as an interfacial tension coefficient. The surface area $\mathcal{A}$, 


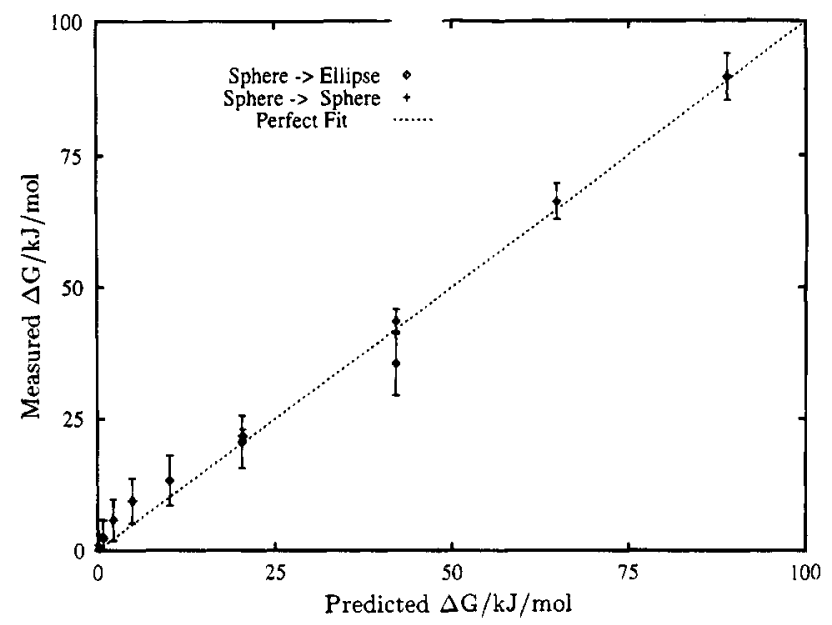

Figure 4. Predicted versus measured values of the free energy change for the systems studied.

Sphere -> Ellipsoid Sphere $\rightarrow$ Sphere $\square$

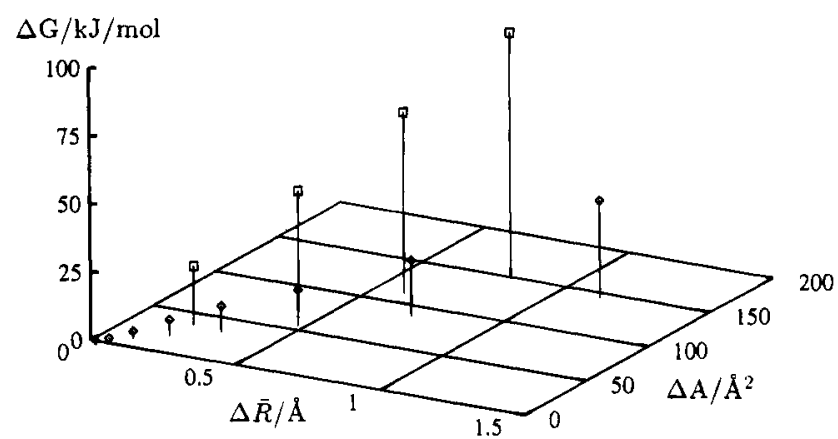

Figure 5. 2D plot of the free energy change with respect to curvature and area.

and the curvature of that area $1 / \vec{R}$, have yet to be specified. A third term involving pressure-volume work is negligible for the dimensions of the systems studied here; e.g., a volume change of $500 \AA^{3}$ at 1 atm pressure gives rise to a $P \Delta V$ contribution of $0.03 \mathrm{~kJ} / \mathrm{mol}$. Of course, a pressure-volume term does not appear in the sphere deformation experiment as it is volume conserving. If the dimensions of the system were macroscopically large, and the deformation is still volume conserving, the area term will become the dominant contribution. The change in free energy, $\Delta G$, with respect to the initial spherical volume with $\sigma_{0}=6.45 \AA$ involves three parameters, $\delta r, \lambda$, and $\gamma$, where $\delta r$ defines the dividing surface and thus the location of the surface area relative to the van der Waals area,

$$
\Delta G(\bar{R}(\delta r), \mathcal{A}(\delta r))=\lambda \Delta \bar{R}(\delta r)+\gamma \Delta \mathcal{A}(\delta r)
$$

A fit of this equation to both sets of data yields $\delta r=0.665 \AA$, $\lambda=-116.5 \mathrm{~kJ} /(\mathrm{mol} \AA)$, and $\gamma=1.205 \mathrm{~kJ} /\left(\mathrm{mol} \AA^{2}\right.$ ) (or equivalently $\gamma=1.9 \times 10^{2} \mathrm{dyn} / \mathrm{cm}$ ) for our model system. The predicted versus the measured values of the free energy change are displayed in Figure 4. The free energy change is also given as a $2 D$ plot of the effective curvature of radius and area in Figure 5. A more precise description of $\Delta G(\bar{R}, \mathcal{A})$ would include an integration over the surface where each surface element would pick up the local curvature contribution. ${ }^{5}$ It is also possible to introduce corrections that would involve the eccentricity explicitly, ${ }^{42}$ but, given the uncertainties of the collected data and the ability of eq 23 to reproduce the observations, further refinement was not deemed expedient. In the spherical case where the curvature is the inverse of the radius, we can write the free energy change as ${ }^{3}$

$$
\Delta G(\bar{R}, \mathscr{A})=\gamma \Delta[\mathcal{M}\{1-a / R\}]
$$

where $a$ is a parameter on the order of the molecular size of the curved object. From the fitted data, $a$ is then found to be $7.7 \AA$, which is comparable to the cavity size of $6.45+\delta r=$ $7.1 \AA$.

The interfacial surface tension between water and the normal alkanes, $n$-hexane through $n$-decane, is $50 \mathrm{dyn} / \mathrm{cm}$ at room temperature..$^{43}$ Our predicted values are thus too large. One should keep in mind that our calculation does not address two contributions, the van der Waals attraction between the solute and water and the free energy penalty of rearranging the solute itself from a sphere to a pancake. In principle, the latter part could be estimated by calculating the free energy change of a set of chain molecules enclosed by a constraining potential mimicking the shape changes of the elliptical contortion. The role of attractions between the solute and water can be gauged by looking at the progression of the experimentally determined interfacial tensions, ranging from $70 \mathrm{dyn} / \mathrm{cm}$ for the liquid water/ vapor through $50 \mathrm{dyn} / \mathrm{cm}$ for the water/oil interface, to the low value of $8.5 \mathrm{dyn} / \mathrm{cm}$ for a water 1 -octanol system. ${ }^{43}$ Thus, attractive forces cause a lowering of the interfacial tension, but even with a $30 \%$ reduction of the interfacial tension our model predictions cannot be said to be quantitative.

Widom $^{24}$ and Stillinger ${ }^{25}$ have suggested that the interface between the water solution and a hydrophobic object is more or less characterized by a thin vapor film, and thus the interfacial tension should be determined by the same factors that govern the interfacial tension between water and its vapor. The measured liquid-vapor interfacial tension for our model potential has been determined to $\mathrm{be}^{23} \gamma_{\mathrm{RER}(\mathrm{par})}=1.0 \times 10^{2}$ $\mathrm{dyn} / \mathrm{cm}$, which is very different from the solute-water $\gamma$ value of $1.9 \times 10^{2} \mathrm{dyn} / \mathrm{cm}$ calculated above. This discrepancy may result from differences in solvent structure around spherical and ellipsoidal solute cavities or from the observation that these solvent water molecules cannot be described by macroscopic surface thermodynamic arguments.

Although it is possible to fit the data with a macroscopic model, such a fit does not necessarily validate the connection between microscopic and macroscopic properties. Indeed for the small particles treated here, virtually any power of any reasonably defined solute radius should yield a linear relationship between the free energy change and that the radius, for the deformation paths followed here.

\section{Characterization of the Solvent}

We investigate the distribution of water molecules in annular ellipsoidal shells around the oblate solute molecule by determining the number of water molecules, $\Delta N$, in a shell of annular volume $\Delta v$,

$$
\Delta v=\frac{4 \pi}{3} \frac{\sigma_{11}}{\sigma_{\perp}} r^{2} \Delta r
$$

where $r$ is the distance from the center of the oblate solute to the beginning of the shell along a direction parallel to one of the $\sigma_{\perp}$ axes, and $\Delta r$ is the thickness of the shell. In essence each shell is labeled by $r$, the distance of closest approach of its inner surface to the center of the solvent excluded volume of the ellipsoidal solute. We then define the relative density of molecules in the shell, 


$$
P(r) \equiv \frac{\varrho(r)}{\varrho}=\frac{\Delta N}{\varrho \Delta v}
$$

where $\varrho$ is the bulk density. $P(r)$ gives the relative water density in a shell located at distance $r$ from the ellipsoidal surface. For a spherical solute, $\sigma_{\perp}=\sigma_{\|}, P(r)$ reduces to the ordinary radial distribution function $g^{(2)}(r)$.

The distributions of water molecules around different hydrophobic solute particles are given in Figure 6. As expected, there is a slight enhancement of water correlations just outside the surface, enabling us to distinguish one hydration shell commensurate with hydrophobic solvation of other nonpolar molecules. $^{44}$ In contrast, ${ }^{45}$ no discernible water structuring was found around a hydrophobic cylinder composed of individual methane molecules that were allowed to rearrange themselves continuously and thus form a more malleable hydrophobic surface. From the distributions in Figure 6, there appears to be a close resemblance between the features of both the spherical and elliptical hydration shells. The liquid-vapor interface of pure water is given for comparison. At the vapor interface there is no density enhancement, indicating a less strained interface, qualitatively similar to the water/hydrocarbon interface made up of individually unconstrained methane molecules. ${ }^{45}$

The interpretation of the coefficient multiplying the area change in eq 23 as an interfacial tension is consistent with the value $\left(1.9 \times 10^{2} \mathrm{dyn} / \mathrm{cm}\right)$ being larger than for the water/vapor system $\left(1.0 \times 10^{2} \mathrm{dyn} / \mathrm{cm}\right)$. It is energetically more costly to create an ordered interface, seen in the enhanced water densities around the solutes in Figure 6 , than to create the more structurally relaxed interface between liquid and vapor. That both types of interfaces shown in Figure 6 should have the same interfacial tension cannot be justified from a microscopic point of view.

In Figure 7 the solute-water radial distribution functions are given. Here we see a gradual and uniform shift of the entire distribution as a function of increasing solute size. This increase is also reflected in the total volume of the system, given in Table 4. In the sphere growth experiments, at constant pressure, the increase of the solute size leads to an increase in the total system volume. The increase in the total volume roughly follows that of the volume increase of the solute. At the largest radius investigated, the volume increase of the solute corresponds to about 16 water molecules. As expected, there is no significant increase of the total system volume upon deformation of the sphere into an ellipsoid. The fluctuation of the volume, $\sim 60$ $\AA^{3}$, is comparable to the volume of two water molecules.

The number of water molecules associated with the first shell, defined by $\sigma+\delta r$, is given in Table 5 for the different shapes of the solute. Although there is a consistent increase of water molecules associated with the shell, this is only clearly manifested for the systems with large area changes. One water molecule with an effective size of $\sigma=2.8$, i.e., corresponding to the first peak in the oxygen-oxygen pair correlation function of pure water, would project an area of $\pi \sigma^{2} \sim 25 \AA^{2}$ onto a flat surface. Only for ellipsoids of large eccentricity will the change in area be large enough to accommodate additional water molecules into the shell. The roughly constant numbers of water molecules in close contact with the solute is also reflected in the similar values of the potential energy between the solute and the surrounding water molecules given in Table 4. Consequently, the free energy change per shell molecule, $\Delta G$ / $n_{\text {shell }}$, is not constant but depends on the characteristics of the solute surface.

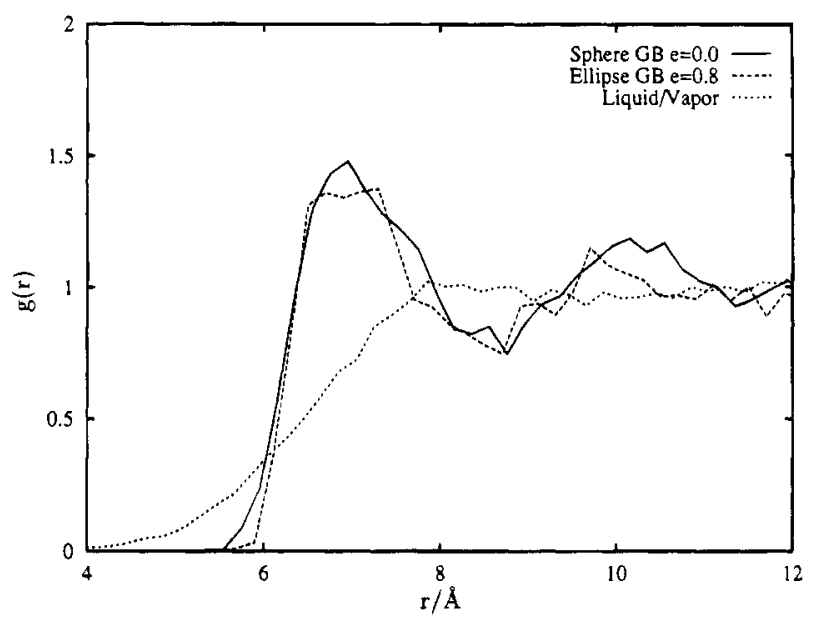

Figure 6. Solute-water radial distribution functions for the RER(pair) water model corresponding to the sphere, $e=0.0$, and for the most extended oblate ellipse, $e=-0.8$. The vapor/liquid-water interface is also indicated.

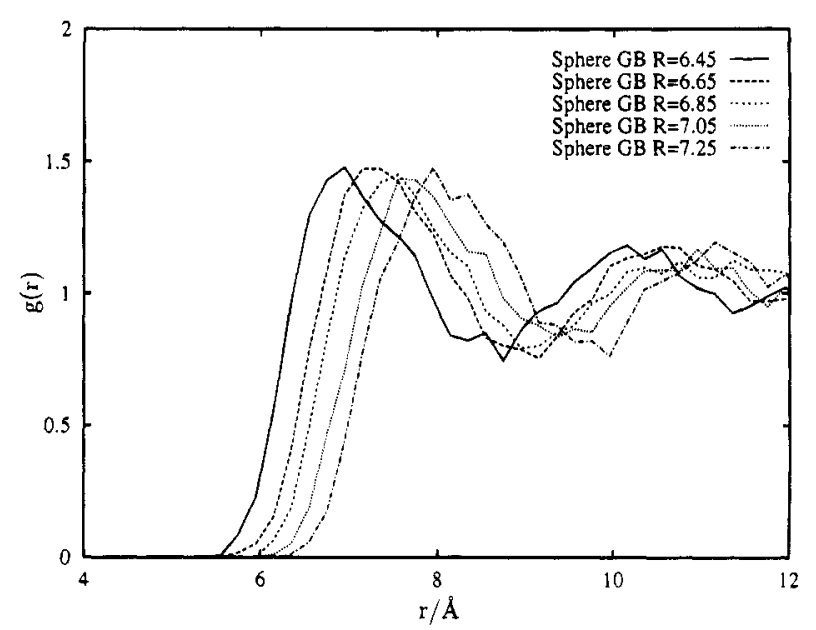

Figure 7. Solute-water radial distribution functions for the sphere growth systems.

TABLE 4: Properties of the System

\begin{tabular}{|c|c|c|c|}
\hline$e$ & $\bar{R}, \AA$ & $V_{\text {system }}, \AA^{3}$ & $U_{\mathrm{sw}}, \mathrm{kJ} / \mathrm{mol}$ \\
\hline \multicolumn{4}{|c|}{ Sphere Deformation into an Ellipse } \\
\hline 0.0 & 6.450 & $28680 \pm 60$ & $63 \pm 4$ \\
\hline-0.1 & 6.461 & $28690 \pm 60$ & $61 \pm 5$ \\
\hline-0.2 & 6.496 & $28660 \pm 60$ & $60 \pm 4$ \\
\hline-0.3 & 6.555 & $28660 \pm 70$ & $59 \pm 4$ \\
\hline-0.4 & 6.643 & $28640 \pm 70$ & $61 \pm 4$ \\
\hline-0.5 & 6.768 & $28630 \pm 70$ & $64 \pm 4$ \\
\hline-0.6 & 6.945 & $28670 \pm 60$ & $55 \pm 4$ \\
\hline-0.7 & 7.205 & $28690 \pm 70$ & $61 \pm 5$ \\
\hline-0.8 & 7.623 & $28650 \pm 60$ & $62 \pm 5$ \\
\hline \multicolumn{4}{|c|}{ Sphere Growth } \\
\hline 0.0 & 6.450 & $28690 \pm 60$ & $63 \pm 4$ \\
\hline 0.0 & 6.650 & $28750 \pm 60$ & $59 \pm 4$ \\
\hline 0.0 & 6.850 & $28890 \pm 50$ & $63 \pm 4$ \\
\hline 0.0 & 7.050 & $29070 \pm 60$ & $64 \pm 4$ \\
\hline 0.0 & 7.250 & $29130 \pm 70$ & $69 \pm 4$ \\
\hline
\end{tabular}

\section{Conclusion}

We have investigated the properties of a water-solute system in which we deformed a hydrophobic solute from a sphere into an essentially flat oblate ellipsoid of the same volume, corresponding to a disk of molecules. A single-site water-solute interaction model based on the Gay-Berne potential was constructed with a soft repulsive core. The interaction depends on the relative orientation of a water molecule with respect to 
TABLE 5: Interfacial Water Properties

\begin{tabular}{rccccc}
\hline$e$ & $\bar{R}, \AA$ & $N_{\text {shell }}$ & $e$ & $\bar{R}, \AA$ & $N_{\text {shell }}$ \\
\hline \multicolumn{5}{c}{ Sphere Deformation into an Ellipse } \\
0.0 & 6.450 & $23.1 \pm 0.3$ & -0.5 & 6.768 & $25.9 \pm 0.3$ \\
-0.1 & 6.461 & $22.2 \pm 0.3$ & -0.6 & 6.945 & $22.8 \pm 0.3$ \\
-0.2 & 6.496 & $22.8 \pm 0.3$ & -0.7 & 7.205 & $25.2 \pm 0.3$ \\
-0.3 & 6.555 & $21.3 \pm 0.3$ & -0.8 & 7.623 & $28.0 \pm 0.3$ \\
-0.4 & 6.643 & $23.2 \pm 0.3$ & & \\
0 \\
0.0 & 6.450 & $23.1 \pm 0.3$ & 0.0 & 7.050 & $24.0 \pm 0.3$ \\
0.0 & 6.650 & $22.6 \pm 0.3$ & 0.0 & 7.250 & $24.9 \pm 0.3$ \\
0.0 & 6.850 & $23.7 \pm 0.3$ & &
\end{tabular}

the major axis of the solute. The solute volume is preserved during the deformation because the deformation conserves the solute volume. The solute-induced water cavity is smoothly deformed between the two extremes by changing a single parameter, namely, the eccentricity $e$. The core of the most deformed solute corresponds to the repulsion a water molecule experiences as it approaches a real methane sheet. The deformation of the sphere into an oblate ellipsoid was also compared to a system where a sphere was grown radially into a set of successively large spheres.

To interpret the results, the free energy change was expanded as a linear function of the radius of curvature and area of the solute-water interface. This required the definition of the location of the surface to be used as the proper interface. In the study of cavity growth of Postma et al..$^{20}$ a surface corresponding to the van der Waals area was chosen when fitting their results to the scaled particle results. From our results we could not make a consistent interpretation using this surface. Instead, the location of the interfacial dividing surface was found by fitting it to be at the first maximum of the solute-water pair-correlation function. A large free energy curvature dependence was detected. If we interpret the coefficient multiplying the change in area as an interfacial tension, we obtain a surface tension of $\gamma=1.9 \times 10^{2} \mathrm{dyn} / \mathrm{cm}$. This value is large compared to the gas-liquid surface tension. $\gamma=1.0 \times 10^{2} \mathrm{dyn} / \mathrm{cm}$ for our water/vapor model. It should be noted that the gas-liquid surface tension found from the RER model is large compared to the experimental value of $\gamma=0.75 \times 10^{2} \mathrm{dyn} / \mathrm{cm}$. The force field for RER water does not contain three-body dispersion interactions or polarizability. Obviously, any solute-water interfacial tension determined using the RER model will also disagree with experiment for the same reasons. The important thing to note here is the internal consistency. We compare the coefficients for the same model of water and draw conclusions from their differences.

From a microscopic point of view the free energy penalty of maintaining water molecules at the interface is intimately connected to how well such molecule can accommodate to an unfavorable environment. The introduction of the inert solute prevents an interfacial water molecule from forming an optimal number of hydrogen bonds. In comparing the two processes of either deforming a sphere into a ellipsoid or growing a sphere into a larger sphere, we note that we are continuously changing the relative orientations of interfacial waters and consequently also their interaction energy with each other as well as with the surrounding water molecules in the bulk. These properties cannot generally be reduced to a function of curvature and area depending on macroscopically measured coefficients without taking into account the molecular nature of the interface. Indeed, for nonvolume conserving systems approaching the macroscopic limit the dominating term in the free energy expression would have to be a $P \Delta V$ term. Still it would be of interest to use the free energy values calculated here together with the curvature corrected area procedure developed by Honig and co-workers ${ }^{4,5}$ to check for consistency.

This study calls into question estimates of the free energy of hydrophobic hydration based on multiplying the solvent accessible surface area by a constant free energy per unit area because it shows, for the simple cases of the expansion and the deformation of a sphere, that different factors of free energy per unit area are required for objects of different curvature. There is clearly an important shape dependence not accounted for by this approximation. Moreover, we have found that a hydrophobic oblate ellipsoid will be more soluble in water than a hydrophobic sphere of the same surface area. We believe that this conclusion is robust against different models of water. Although the shape dependence of the free energy can be formulated, to a first approximation, in terms of a curvature dependence, we do not believe that such a macroscopic concept may be generally applicable to more realistic solute-solvent interactions.

Acknowledgment. This work was supported by a grant from the National Institutes of Health (GM43340-01A1) and was done at the NIH Biotechnology Research Center at Columbia University. We acknowledge that the simulations were carried out on a network of IBM RISC/6000 workstations purchased on an NSF department instrument grant. We thank Profs. L. R. Pratt and Barry Honig for useful discussions.

\section{References and Notes}

(1) Tanford, C. The Hydrophobic Effect; Wiley: New York, 1973.

(2) Richards, F. M. Annu. Rev. Biophys. Bioeng. 1977, 6, 151.

(3) Buff, F. P. The sperical interface. I. Thermodynamics. J. Chem. Phys. 1951, 19, 1591.

(4) Sharp, K. A.; Nicholls, A.; Fine, R. F.; Honig, B. Reconciling the magnitude of the microscopic and macroscopic hydrophobic effects. Science 1991, 252, 106-109.

(5) Nicholls, A.; Sharp, K. A.; Honig, B. Protein folding and association: Insights from the interfacial and thermodynamic properties of hydrocarbons. Proteins: Struct., Funct., Genet. 1991, 11, 281-296.

(6) Tunon, I.; Silla, E.; Pascual-Ahuir, J. L. Moleculat surface area and the hydrophobic effect. Protein Eng. 1992, 5, 715-716.

(7) Ben-Naim, A.; Mazo, R. M. Size dependence of the solvation free energies of large solutes. J. Phys. Chem. 1993, 97, 10829-10834.

(8) Ben-Naim, A. Solvation: from small to macro molecules. Curr. Opin. Struct. Biol. 1994, 4, 264-268.

(9) Giesen, D. J.; Cramer, C. J.; Truhlar, D. G. Entropic contributions to free energies of solvation. J. Phys. Chem. 1994, 98, 4141-4147.

(10) Pangali, C.; Rao, M.; Berne, B. J. Hydrophobic hydration around a pair of apolar species in water. J. Chem. Phys. 1979, 71, 2982-2990.

(11) Okazaki, S.; Nakanishi, K.; Touhara, H.; Watanabe, N.; Adachi, Y. A Monte Carlo study of the size dependence in hydrophobic hydration. J. Chem. Phys. 1981, 74, 5863-5871.

(12) Zichi, D. A.; Rossky, P. J. The equilibrium solvation structure for the solvent separated hydrophobic bond. J. Chem. Phys. 1985, 83, 797808 .

(13) Zichi, D. A.; Rossky, P. J. Solvent molecular dynamics in regions of hydrophobic hydration. J. Chem. Phys. 1986, 84, 2814-2822.

(14) Tanaka, H. Integral equation and Monte Carlo study on hydrophobic effects. Size dependence of apolar solutes on solute-solute interactions and structure of water. J. Chem. Phys. 1987, 86, 1512-1520.

(15) Lee, C. Y.; McCammon, J. A.; Rossky, P. J. The structure of liquid water at an extended hydrophobic surface. J. Chem. Phys. 1984, 80, 44484455 .

(16) Valleau, J. P.; Gardner, A. A. Water-like particles at surfaces. I. The uncharged, unpolarizable surface. J. Chem. Phys. 1987, 86, 41624170 .

(17) Wallqvist, A.: Berne, B. J. Hydrophobic interaction between a methane molecule and a paraffin wall in liquid water. Chem. Phys. Lett. $1988,145,26-32$.

(18) Wallqvist, A. Polarizable water at a hydrophobic wall. Chem. Phys. Lett. 1990, 165, 437-442.

(19) Lee, S. H.; Rossky, P. J. A comparison of the structure and dynamics of liquid water at hydrophobic and hydrophilic surfaces-a molecular dynamics simulation study. J. Chem. Phys. 1994, 100, 33343345. 
(20) Postma, J. P. M.; Berendsen, H. J. C.; Haak, J. R. Thermodynamics of cavity formation in water. Faraday Symp. Chem. Soc. 1982, 17, 5567.

(21) Jorgensen, W. L.; Blake, J. F.; Buckner, J. K. Free energy of TIP4P water and the free energies of hydration of $\mathrm{CH}_{4}$ and $\mathrm{Cl}^{-}$from statistical perturbation theory. Chem. Phys. 1989, 129, 193-200.

(22) Wallqvist, A.; Berne, B. J. Effective potentials for liquid water using polarizable and nonpolarizable models. J. Phys. Chem. 1993, 97, 1384113851.

(23) Wallqvist, A.; Berne, B. J. Liquid-vapor interface of polarizable and nonpolarizable water. Manuscript in preparation.

(24) Widom, B. J. Chem. Phys. 1965, 43, 3829.

(25) Stillinger, F. H. Structure in aqueous solutions of nonpolar solutes from the standpoint of scaled particle theory. J. Solution Chem. 1973, 2 , $141-158$.

(26) Berne, B. J.; Pechukas, P. Gaussian model potentials for molecular interactions. J. Chem. Phys. 1972, 56, 4213-4216.

(27) Gay, J. G.; Berne, B. J. Modification of the overlap potential to mimic a linear site-site potential. J. Chem. Phys. 1981, 74, 3316-3319.

(28) Barker, J. A.; Henderson, D. What is 'liquid'? Understanding the states of matter. Rev. Mod. Phys. 1976, 48, 587-671.

(29) Zwanzig, R. W. J. Chem. Phys. 1954, 22, 1420.

(30) Beveridge, D. L.; DiCapua, F. M. Free energy via molecular simulations: Applications to chemical and biomolecular systems. Annu. Rev. Biophys. Biophys. Chem. 1989, 18, 431-492.

(31) Smith, D. E.; Haymet, A. D. J. Free energy, entropy, and internal energy of hydrophobic interactions: Computer simulations. J. Chem. Phys. 1993, 98, 6445-6454.

(32) Berendsen, H. J. C.; Grigera, J. R.; Straatsma, T. P. The missing term in effective pair potentials. J. Phys. Chem. 1987, 91, 6269-6271.

(33) Watanabe, K.; Klein, M. L. Effective pair potentials and the properties of water. Chem. Phys, 1989, 131, 157-167.

(34) Andersen, H. C. Rattle: A 'velocity' version of the shake algorithm for molecular dynamics calculations. J. Comput. Phys. 1983, 52, 24-34. (35) Swope, W. C.; Andersen, H. C.; Berens, P. H.; Wilson, K. R. A computer simulation method for the calculation of equilibrium constants for the formation of physical clusters of molecules: Applications to small water clusters. J. Chem. Phys. 1982, 76, 637-649.

(36) Wallgvist, A.; Teleman, O. Properties of flexible water models. Mol. Phys. 1991, 74, 515-533.

(37) Linse, P. Stacked or T-shaped benzene dimers in aqueous solution? A molecular dynamics study. J. Am. Chem. Soc. 1992, 114, 4366-4373.

(38) Gerstein, M.; Lynden-Bell, R. M. Simulation of water around a model protein helix. 1. Two dimension projections of solvent structure. J. Phys. Chem. 1993, 97, 2982-2990.

(39) Gerstein, M.; Lynden-Bell, R. M. Simulation of water around a model protein helix. 2. The relative contributions of packing, hydrophobicity, and hydrogen bonding. J. Phys. Chem. 1993, 97, 2991-2999.

(40) Gerstein, M.; Lynden-Bell, R. M. What is the natural boundary of a protein in solution? J Mol. Biol. 1993, 230, 641-650.

(41) Reiss, H. Scaled particle methods in the statistical thermodynamics of fluids. Adv. Chem. Phys. 1965, 9, 1-84.

(42) Kirkwood, J. G.; Buff, F. P. The statistical mechanical theory of surface tension. J. Chem. Phys. 1949, 17, 338-343.

(43) Ross, S.; Morrison, I. D. Colloidal Systems and Interfaces; Wiley: New York, 1988

(44) Rapaport, D. C.; Scheraga, H. A. Hydration of inert solutes. A molecular dynamics study. J. Phys. Chem. 1982, 86, 873-880.

(45) Wallqvist, A. Molecular dynamics study of a hydrophobic aggregate in an aqueous solution of methane. J. Phys. Chem. 1991, 95, 8921-8927.

JP9420640 